\title{
Early and long-term morbidity after total laryngopharyngectomy
}

\author{
Stijn Keereweer · Johannes H. W. de Wilt • \\ Aniel Sewnaik · Cees A. Meeuwis · Hugo W. Tilanus • \\ Jeroen D. F. Kerrebijn
}

Received: 7 January 2010/Accepted: 26 March 2010/Published online: 7 April 2010

(C) The Author(s) 2010. This article is published with open access at Springerlink.com

\begin{abstract}
To determine the early and long-term morbidity of patients treated with a total laryngopharyngectomy and reconstruction using a jejunum interposition or gastric pull-up procedure. It is a retrospective study; and it is conducted in tertiairy referral center. Sixty-three patients were included in whom 70 reconstructions were performed (51 jejunum interpositions and 19 gastric pull-up procedures) between 1990 and 2007. The studied parameters were success rate of the reconstruction, early and long-term complication rate, and functional outcome including quality of life. Subjective quality of life analysis was determined by two questionnaires: the EORTC Quality of Life Questionnaire (QLQ)-C30 Dutch version 3.0, and the EORTC-Head and Neck ( $\mathrm{H} \& \mathrm{~N} 35)$. The success rates were 84 and $74 \%$, respectively. The procedures were associated with a high complication rate $(63 \%$ after
\end{abstract}

Presented at The Netherlands Society of Otorhinolaryngology meeting, November 2008 and the Annual Meeting of the American Academy of Otolaryngology Head and Neck Surgery, October 2009.

S. Keereweer $(\bowtie) \cdot$ A. Sewnaik · C. A. Meeuwis .

J. D. F. Kerrebijn

Department of Head and Neck Surgery, Erasmus University

Medical Center, Daniel den Hoed Cancer Center,

301 Groene Hilledijk, 3075 EA Rotterdam, The Netherlands

e-mail: s.keereweer@erasmusmc.nl

J. H. W. de Wilt

Department of Surgical Oncology, Radboud University

Nijmegen Medical Center, Nijmegen, The Netherlands

H. W. Tilanus

Department of Surgical Oncology, Erasmus University Medical Center, Daniel den Hoed Cancer Center,

Rotterdam, The Netherlands jejunum interposition and $89 \%$ after gastric pull-up), and a lengthy rehabilitation. Surviving patients were found to have a good long-term quality of life. Complete oral intake was achieved in $97 \%$, and speech rehabilitation in $95 \%$. These procedures are associated with significant morbidity, high complication rates, lengthy rehabilitation, but a good long-term quality of life.

Keywords Jejunum interposition · Gastric pull-up · Laryngopharyngectomy · Functional results .

Reconstruction

\section{Introduction}

Extensive malignant tumors of the laryngopharyngeal region and the cervical oesophagus are associated with a poor prognosis, despite various treatment modalities. Because symptoms of this disease occur in a late stage, most patients (71-86\%) are diagnosed with advanced stage disease (III-IV), which is associated with a 5-year survival rate of $15-47 \%[1,2]$.

The morbidity of a total laryngopharyngectomy, especially impaired speech and swallowing function, significantly influences the daily quality of life of the patients. These problems, together with the persisting low survival rates, have stimulated the development of other treatment regimens. Concomitant chemoradiotherapy has been shown to allow conservation of the larynx without any negative effect on survival [3, 4]. However, when salvage surgery after this treatment is required, the toxicity of these previous therapies limits the population of suitable patients. When ablative surgery is considered the best therapeutic option, reconstructive surgeons are confronted with the challenge to cover the defect and achieve sufficient 
functional results with regard to swallowing and voice restoration, while minimizing morbidity in a high-risk population.

In our institution, two reconstructive modalities have become the accepted standard reconstructive techniques after surgical ablation of tumors in the laryngopharyngeal region and cervical oesophagus. For the reconstruction of circumferential defects of the hypopharynx that do not include any part of the oesophagus, jejunum interposition generally is the preferred technique. In case of a lower located lesion, a reconstruction using the gastric pull-up is preferred, especially when the resection extends inferiorly to the thoracic inlet [5-10].

Many surgeons will agree that these procedures are related to significant postoperative morbidity. However, few studies have described the actual extent of early and long-term complications in this difficult to treat group of patients. The aim of this study is to review our results of reconstruction surgery with jejunum interposition or gastric pull-up following total laryngopharyngectomy in terms of success rate, postoperative complications and the restoration of the quality of life, including speech and swallowing functions.

\section{Materials and methods}

This is a retrospective analysis of all patients who underwent a total laryngopharyngectomy with reconstruction using a jejunum interposition or gastric pull-up procedure in the Erasmus Medical Centre between January 1990 and April 2007. The data were collected using the medical records.

A total of 63 patients ( 55 males and 8 females) in whom 70 reconstructions were performed were included with a mean age of 61 years (range 38-81 years). In seven patients, a re-operation was necessary due to postoperative flap failure. The indications for the operations were divided into four categories: primary tumors $(50 \%)$, recurrences (29\%), functional reasons (11\%), or revision operations $(10 \%)$ (Table 1). All patients treated for a primary tumor underwent postoperative radiotherapy. Patients treated for a tumor recurrence had previously been treated with

Table 1 Indication for operation in total no. of operations $(n=70)$

\begin{tabular}{lccc}
\hline Indication & Jejunum interposition & Gastric pull-up & Total \\
\hline Primary tumor & 22 & 13 & 35 \\
Recurrence of tumor & 18 & 2 & 20 \\
Functional & 7 & 1 & 8 \\
Revision operations & 4 & 3 & 7 \\
Total & 51 & 19 & 70 \\
\hline
\end{tabular}

radiotherapy $(n=5)$, chemoradiation $(n=5)$, surgery $(n=1)$, or any other combination $(n=9)$. The American Society of Anesthesiologists (ASA) classification was used as an indication for co-morbidity. Of all patients, $4 \%$ was ASA I (no known systemic disease), $41 \%$ ASA II (mild systemic disease), and 55\% ASA III (severe systemic disease).

\section{Surgical procedures}

The choice of the reconstructive method was made by the attending surgeon. In general, a gastric pull-up procedure was performed for more inferiorly localized tumors, extending into the cervical oesophagus. Defects of superiorly localized tumors were reconstructed with a jejunum interposition. Postoperative nutrition was maintained by placement of a feeding jejunostomy.

\section{Study outcomes}

The studied parameters were success rate of the reconstruction, complication rate, and functional outcome including quality of life. The success rate is defined as the percentage of patients with a viable jejunum interposition or gastric pull-up. The reconstructions in patients who died in the direct postoperative period were considered unsuccessful. Complications were scored as early complications ( $<3$ months postoperatively) or late complications ( $>3$ months postoperatively), and were divided into surgical and non-surgical complications. In addition, complications occurring after primary surgery or salvage surgery were reviewed separately.

Swallowing rehabilitation was considered successful when complete oral intake was achieved, discarding the need for enteral supplementation. Speech rehabilitation was considered successful when patients were able to communicate by speech. No assessment of voice quality was performed. All patients had professional assistance in speech rehabilitation. Patients alive at the time of the study (October 2007-January 2008) received two questionnaires by mail: the EORTC Quality of Life Questionnaire (QLQ)C30 Dutch version 3.0 [11], and the EORTC-Head \& Neck (H \& 35) [12].

The EORTC QLQ-C30 [11] consists of five functional scales: physical (5 items), role (2 items), emotional (4 items), cognitive (2 items), and global health status ( 2 items); 3 symptom scales: fatigue ( 3 items), nausea/ vomiting ( 2 items), and pain ( 2 items); and six single items: dyspnoea, insomnia, appetite loss, constipation, diarrhea, and financial difficulties. In total, there are 30 questions.

The EORTC QLQ-H \& N 35 [12] is a diagnosis-specific questionnaire for patients with head and neck cancer. It consists of 35 questions concerning problems that are 
Table 2 TNM stage of patients treated for primary or recurrent tumor $(n=55)$

\begin{tabular}{crrrrr}
\hline & T2 & T3 & T4 & Tx & Total \\
\hline M0 & & & & & \\
N0 & 12 & 6 & 9 & 3 & 30 \\
N1 & 2 & 4 & 4 & 0 & 10 \\
N2a & 1 & 0 & 1 & 0 & 2 \\
N2b & 2 & 2 & 5 & 0 & 9 \\
N2c & 1 & 0 & 2 & 0 & 3 \\
N3 & 0 & 0 & 1 & 0 & 1 \\
Total & 18 & 12 & 22 & 3 & 55 \\
\hline
\end{tabular}

attributable to the head and neck cancer and treatment morbidity. Because a high scale score represents a higher response level, high scores for functional items or global health status represent high levels of functioning. In contrast, a high score for a symptom item represents a high level of symptomatology or problems.

Statistical analysis was performed using SPSS version 15 statistical software. Categorical data were analyzed using the Pearson $\chi^{2}$ test.

\section{Results}

Fifty-one jejunum interpositions and 19 gastric pull-up procedures were performed. The median hospital stay for the whole group was 30 days (range 5-126 days). Indications of operation are summarized in Table 1. Of the eight patients operated for functional reasons, four operations were performed due to fibrosis as a result of chemoradiation (3), or radiotherapy (1). Four operations were done because of stenosis or persisting fistulas. In the patients treated for a primary or recurrent tumor, the tumor was located in the hypopharynx $(n=40)$, larynx $(n=5)$, cervical oesophagus $(n=8)$, or oropharynx with extension into the postcricoid region $(n=2)$. The TNM classifications of these patients are presented in Table 2.

\section{Success rate}

The success rates of the 47 jejunum interpositions and 16 gastric pull-up procedures in 63 patients were 85 and $69 \%$, respectively (Fig. 1). When including the seven revision operations, of which six were successful, the success rates were 84 (43/51) and 74\% (14/19), respectively. The postoperative mortality (in-hospital mortality) for patients undergoing total laryngopharyngectomy and reconstruction with jejunum interposition was $4 \%(n=2)$, while this was $16 \%$ for patients reconstructed with a gastric pull-up $(n=3)$. In the jejunum interposition group, one patient died as result of an ileus, resulting in a septic peritonitis. The second patient died of an acute hemorrhage from the operation wound in the neck 2 days postoperatively. In the gastric pull-up group, one patient died of respiratory failure combined with conduit necrosis, one patient died of postoperative mediastinal hemorrhage, and in one patient a carotid blow-out occurred.

\section{Postoperative complications}

Postoperative complications of the jejunum interposition and gastric pull-up groups are summarized in Table 3 . In the jejunum interposition group, $63 \%$ of the patients had one or

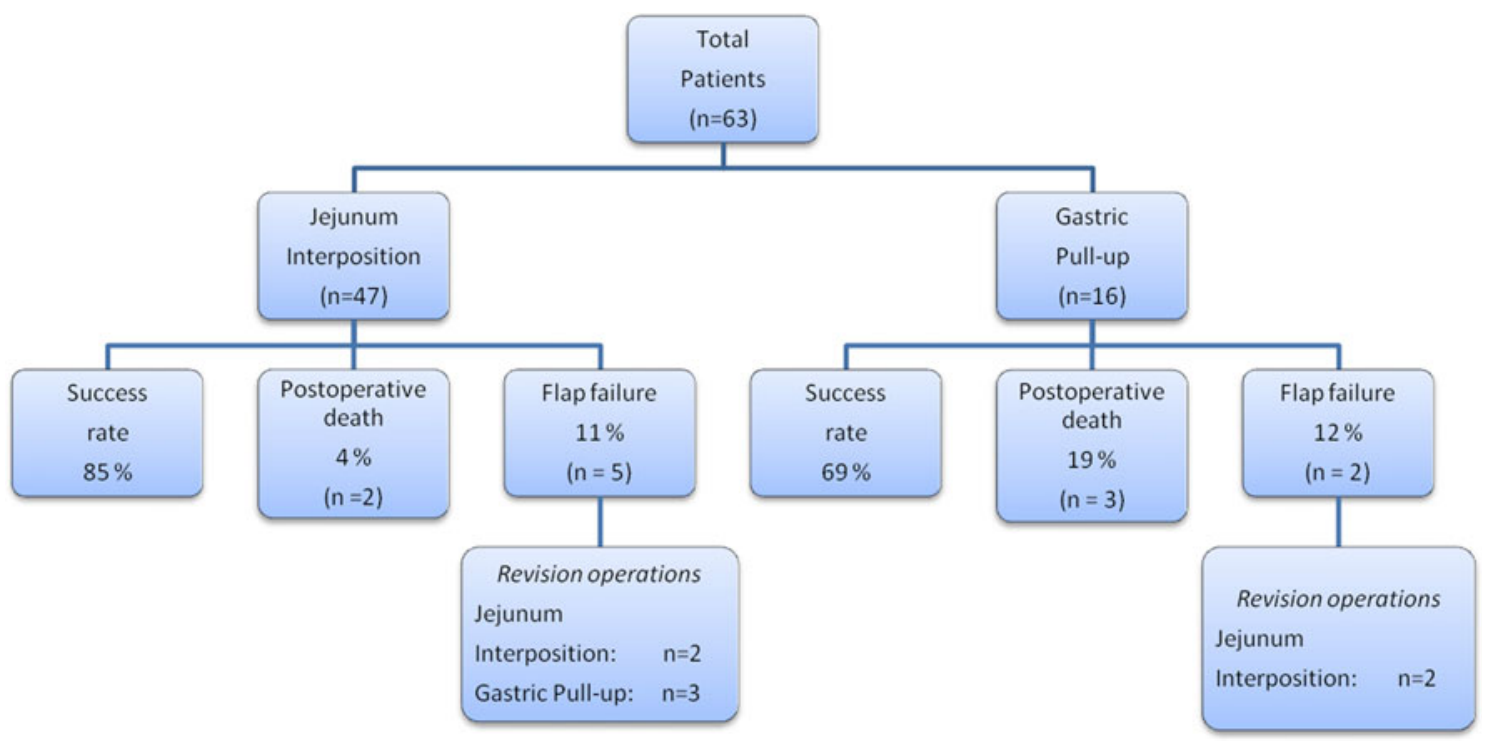

Fig. 1 Success rate of jejunum interposition and gastric pull-up, showing 70 procedures performed in 63 patients 
Table 3 Postoperative surgical and non-surgical complications ( $<3$ months)

\begin{tabular}{|c|c|c|c|}
\hline Complications & Jejunum interposition $(n=51)$ & Gastric pull-up $(n=19)$ & Surgical intervention \\
\hline \multicolumn{4}{|l|}{ Surgical complications } \\
\hline Postoperative mortality & 2 & 3 & - \\
\hline Flap failure & 4 & 2 & $100 \%$ \\
\hline Fistula & 18 & 10 & $39 \%$ \\
\hline Hemorrhage neck & 7 & 2 & $89 \%$ \\
\hline Dehiscence after laparotomy & 2 & 2 & $100 \%$ \\
\hline Wound dehiscence & 1 & 1 & - \\
\hline Ileus & 2 & - & $100 \%$ \\
\hline Horner's syndrome & - & 1 & - \\
\hline Mediastinitis & - & 1 & - \\
\hline Sepsis (due to intestinal necrosis) & 1 & - & $100 \%$ \\
\hline \multicolumn{4}{|l|}{ Non-surgical complications } \\
\hline Pneumonia & 4 & 5 & - \\
\hline Severe depression & 1 & 2 & - \\
\hline Hemiparesis & 1 & - & - \\
\hline Tracheitis & - & 1 & - \\
\hline Cholecystitis & 1 & - & - \\
\hline Pyloric spasm & 1 & - & $100 \%$ \\
\hline Epileptic insults & - & 1 & - \\
\hline Patients with one or more complications & $63 \%(32 / 51)$ & $89 \%(17 / 19)$ & \\
\hline
\end{tabular}

The percentage of cases in which surgical intervention was required is shown

more complications. The average number of complications per patient was 1.4. Eighty percent of these complications were directly surgery related. In the gastric pull-up group, $89 \%$ of the patients had one or more complications. The average number of complications per patient was 1.8 . Seventy-one percent of the complications were directly surgery related. One patient developed a hemiparesis of uncertain etiology (no perioperative hypotension or extensive manipulation of the carotid artery was recorded).

In the group of seven revision operations, four patients were re-operated due to immediate postoperative failure of the jejunum interposition. A new neopharynx was constructed using a second jejunum interposition in two patients and a gastric pull-up in the other two. In two patients with a gastric pull-up, ischemia of the proximal anastomosis occurred after 9 and 21 days. The distal parts of these gastric pull-ups were still vital, allowing the patient to recover from the first operation, before the revision operation was performed after 13 and 21 months, respectively.

\section{Late complications}

The late postoperative complications are displayed in Table 4. In $31 \%$ of all patients, a late complication developed, for which surgical intervention was necessary in $81 \%$ of the cases. Late strictures of the neopharynx were seen in $10 \%$ of the patients treated with a jejunum interposition, and $31 \%$ in the gastric pull-up group. Surgical intervention was required in $40 \%$ of these cases. A stenosis of the tracheostoma developed in $6 \%$ of the jejunum interposition group, and $13 \%$ of the gastric pullup group. In one patient in the group of seven revision operations, the re-operation was required 9 months postoperatively, due to ischemic necrosis of the jejunal flap. The gastro-esophageal tract was restored by the use of a gastric pull-up.

Primary versus recurrent tumors

There was no statistically significant difference in complication rate between the group of patients operated for a primary tumor $(66 \%)$, and patients treated for a recurrent tumor $(80 \%)$. Late complications occurred in $28 \%$ of the patients treated for a primary tumor, compared with $33 \%$ in the recurrent tumor group. This difference was not statistically significant.

Functional outcome and quality of life

\section{Voice rehabilitation}

Owing to five postoperative deaths, speech rehabilitation was attempted in 58 patients. Rehabilitation was achieved in $95 \%$ of the patients, of which $52 \%$ communicated by the use 
Table 4 Late surgical and non-surgical complications ( $>3$ months)

\begin{tabular}{llll}
\hline Complications & Jejunum Interposition $(n=49)^{\mathrm{a}}$ & Gastric pull-up $(n=16)$ & Surgical intervention \\
\hline Surgical complications & & & $100 \%(1 / 1)$ \\
Ischemic necrotic flap & 1 & - & $40 \%(4 / 10)$ \\
Stricture of neopharynx & 5 & 5 & $80 \%(4 / 5)$ \\
Stenosis of tracheostoma & 3 & - & $100 \%(1 / 1)$ \\
Abdominal incisional hernia & 1 & - & $100 \%(1 / 1)$ \\
Abces & 1 & - & $100 \%(1 / 1)$ \\
Intestinal torsion around jejunal fistula & 1 & 1 & - \\
Non-surgical complications & & $50 \%(8 / 16)$ & $81 \%(17 / 21)^{\mathrm{b}}$ \\
$\quad$ Recurring pneumonia & 1 & $24 \%(12 / 49)$ & \\
Patients with one or more complications & & & \\
\hline
\end{tabular}

${ }^{\text {a }}$ Five patients died during the early postoperative period and are therefore not included in this table

b Percentage of surgical intervention for all late complications

of an electrolarynx and $43 \%$ by the use of a Provox ${ }^{\circledR}$ speech button. In three patients (5\%), voice rehabilitation was never achieved and communication was only possible by writing.

\section{Swallowing rehabilitation}

Rehabilitation of swallowing was started at a mean of 18 days (range 3-80 days) postoperatively in the surviving patients. On an average, 64 days (range 4-292 days) were needed to achieve complete oral intake. In all, but two patients $(97 \%)$ complete oral intake was achieved, despite the fact that in seven patients, a revision operation was needed due to flap failure.

\section{Quality of life}

The estimated 5-year overall survival was $25 \%$. A questionnaire was sent to all 21 patients alive at time of the study and 14 were filled in and returned. Twelve patients underwent a jejunum interposition and two patients a gastric pull-up. The indications for operation were primary tumors, recurrent tumors and functional reasons in four, five, and five patients, respectively. Tables 5 and 6 show the results of both questionnaires. Satisfying scores were given for the global health status (mean 81/100) and functional scales (mean 69-94/100), indicating excellent results. The most important issues with negative influence on the quality of life were deterioration of smell and taste senses (mean 60/100), speech problems (mean 48/100), and less sexuality (mean 38/100).

\section{Discussion}

This study on a series of 70 operations in 63 patients with head and neck cancer requiring a total
Table 5 Results from EORTC QLQ-C30 Questionnaire

\begin{tabular}{lc}
\hline Item & Score \\
\hline Global health status & \\
Global quality of life & 81 \\
Functional scales & \\
Physical functioning & 89 \\
Role functioning & 80 \\
Emotional functioning & 80 \\
Cognitive functioning & 94 \\
Social functioning & 69 \\
Symptoms scales & \\
Fatigue & 22 \\
Nausea and vomiting & 5 \\
Pain & 10 \\
Dyspnoea & 15 \\
Insomnia & 13 \\
Appetite loss & 11 \\
Constipation & 3 \\
Diarrhea & 5 \\
Financial difficulties & 19 \\
\hline
\end{tabular}

laryngopharyngectomy with circumferential pharyngeal reconstruction with either jejunum interposition or gastric pull-up shows that perioperative morbidity of both procedures is high. However, the overall success rate of these reconstructions and long-term functional outcome is good in those patients surviving their disease.

Reconstructions with a gastric pull-up seem to have a lower success rate (74 vs. $84 \%)$, higher perioperative mortality (16 vs. $4 \%$ ) and more overall early complications (89 vs. 63\%) than jejunum interposition in our institution. However, these results are biased because a gastric pull-up is mostly used for inferiorly located defects that cannot be reconstructed with jejunum interposition. This is in line 
Table 6 Results from EORTC QLQ-H \& N 35 Questionnaire

\begin{tabular}{lc}
\hline Item & Score \\
\hline Pain & 7 \\
Swallowing & 21 \\
Senses problems & 60 \\
Speech problems & 48 \\
Trouble with social eating & 29 \\
Trouble with social contact & 18 \\
Less sexuality & 38 \\
Teeth & 13 \\
Opening mouth & 22 \\
Dry mouth & 23 \\
Sticky saliva & 26 \\
Coughing & 23 \\
Felt ill & 10 \\
Pain killers & 3 \\
Nutritional supplements & 5 \\
Feeding tube & 8 \\
Weight loss & 8 \\
Weight gain & 5 \\
\hline
\end{tabular}

with the most literature, where a free transfer (i.e. jejunum, colon, tubed radial forearm flap, anterolateral thigh flap) $[6-8,13]$ is preferred in hypopharyngeal defects, and a gastric pull-up is the treatment modality of first choice in esophageal cancer $[5,8,10]$.

To gain an extended overview of the postoperative morbidity of this difficult to treat group of patients, we have described various complications including those not directly related to the surgical procedure itself, making it difficult to compare these numbers to other studies. Most studies only describe wound complications in $27-50 \%$ of the patients [5, 9], which is comparable with $40 \%$ in our overall study population, when only considering the wound complications. Our in-hospital mortality rates of $4 \%$ for jejunum interpositions and $16 \%$ for gastric pull-up procedures are consistent with other reports [5-7, 9, 10, 14, 15].

Pharyngocutaneous fistulas are the most frequently occurring complications associated with these reconstructions in the early postoperative period. The overall fistula rate of $40 \%$ (of which $39 \%$ of the patients needed surgical intervention) in this study compares poorly to a previous report from our institute and other studies [6, 10, 14]. A number of reasons make a comparison of reported fistula rates in different studies difficult. A clear distinction between operations performed for primary versus recurrent tumors or functional (revision) operations is rarely made. This is an important differentiation to make as was shown by a study by Eckardt et al. [15] which included patients operated for both malignant and functional indications (i.e. trauma, congenital defects). Moreover, the indication for surgery has shifted during the past two decades towards more complex patients. The treatment strategies have evolved into organ preservation protocols and consequently, a considerable number of patients were initially treated with (chemo-)radiation. This resulted in a secondary role for surgery in $29 \%$ of the cases when previous therapy (i.e. radiotherapy or chemoradiation) had failed. In addition, $55 \%$ of the patients were classified as ASA III, preventing most of them to be suitable candidates for chemoradiation in the first place. The high rate of pretreated patients together with the significant co-morbidity (96\% ASA II/III) will have largely attributed to the high complication rates. However, due to the large diversity within our patient population (e.g. medical history, pretreatment modalities, disease stage), much larger groups of patients would be required to analyze the contribution of each of these components to the complication rates.

Few studies address long-term complications and morbidity of total laryngopharyngectomy [5, 9]. Twenty-four percent long-term complications for jejunum interpositions and up to $50 \%$ for gastric pull-up, found in our present study, is not insignificant. The majority of long-term complications, requiring surgical intervention in $81 \%$ of cases, consisted of strictures of the neopharynx or of the tracheostoma. The reason for the late developments of these strictures is unclear.

It has been suggested that the trans-esophageal puncture may play a role by compromising the blood flow and progressive fibrosis when performed too proximal to the anastomosis [16]. Theoretically, the jejunum interposition has several advantages. It is likely that mucous secretion in the jejunum interposition will favor the swallowing function. Moreover, a more robust junction will be reached with a mucous-to-mucous anastomosis as compared to a mucous-to-skin anastomosis. Finally, no vertical anastomosis is required when a circular graft is used. However, we have previously reported pouch formation of the jejunum interposition resulting in dysphagia complaints [17], and Murray et al. [18] have suggested that alternative reconstruction methods (tubed radial forearm flap, anterolateral thigh flap) provide better long-term results. Nevertheless, a retrospective study by $\mathrm{Yu}$ et al. [19], comparing the functional outcomes of the jejunum interposition with the anterolateral thigh flap, showed similar complication rates. In conclusion, as long as prospective, randomized controlled trails have not been performed, the optimal reconstructive method for these large circular defects will remain subject to debate.

One of the greatest impacts of total laryngopharyngectomy is the loss of the natural voice. There are numerous means of speech rehabilitation of which the voice prosthesis is currently the most commonly used in our institution. Voice rehabilitation is usually started 10 days 
postoperatively. The use of a Provox ${ }^{\circledR}$ speech button (41\%) or an electrolarynx (52\%) resulted in a total of $95 \%$ of the patients being able to communicate by means of speech. This possibility of direct communication is essential for a sufficient quality of life, which is reflected in satisfying global functional scores (mean 69-100). However, despite the communication capability, significant speech problems were reported (mean 48/100). This most likely reflects the practical and psychological difficulties resulting from the obligatory use of an electrolarynx instead of the natural voice. In our institute, most tracheoesophageal punctures are performed during a secondary procedure, approximately 6 weeks after the first operation. Boscolo-Rizzo et al. [20] found no difference in complication rate between initial or secondary placement of the voice prosthesis. However, we feel that each effect that could provoke wound complications should be avoided. To our knowledge, these are the first results that fully confirm the experience-based presumption of the wide extent of the postoperative morbidity. Complete oral intake, defined as the moment when enteral supplementation is no longer required, was achieved in $97 \%$ of patients, an excellent outcome as compared to the reported $65-81 \%$ in the literature $[7,10,21,22]$. However, despite the lack of need for a feeding tube, in many cases patients are restricted to a soft diet, which could explain the moderate problems in social eating (mean 29/100). Furthermore, late stricture formation was seen in $17 \%$ of the patients, which could explain the mild swallowing problems (mean 21/100). Swallowing rehabilitation started between 3 and 80 days postoperatively with a mean of 18 days. Complete oral intake was on average accomplished after 2 months. These data are rarely reported in the literature, but Oniscu et al. [22] reported satisfactory swallowing of $78 \%$ after 6 months and $81 \%$ after 12 months, suggesting that a rehabilitation period of 2 months should on average be expected. An interesting alternative reconstruction technique was introduced by Schilling et al. [23] who described the use of a fundus rotation gastroplasty resulting in a low complication rate and moderate dysphagia during the first 6 months after surgery which resolved within 1 year. Patients undergoing total laryngopharyngectomy are generally patients with advanced disease, roughly $75 \%$ having stages III or IV disease. Reported 5-year survival rates vary between 11 and $47 \%$ [5, 7, 14, 21, 22]. In this study, the 5 -year survival rate was $25 \%$. Adding the high postoperative complication rate and lengthy rehabilitation period, especially in the salvage setting, it can in some instances be questionable if surgery should at all be offered if there is no (further) alternative treatment available.

However, analysis of the global health score and functional scores of the surviving patients reveal strikingly satisfactory results. Minimal pain was experienced, which is an important factor of gaining an acceptable quality of life. As demonstrated in previous quality of life studies of partial and total laryngectomy [24] and confirmed by our data, significant deterioration in smell and taste functioning does not result in a loss of appetite, contrary to what might be expected. Despite the use of voice prostheses, speech problems are still among the most important complaints during the follow-up of these procedures [24]. No emotional regression was reported. However, some patients did describe a decrease in interest and pleasure regarding sexual activities. With regard to the symptoms, sticky saliva is the most prominently and generally described complaint, followed by fatigue, dry mouth and coughing. Although the assessment of the quality of life was performed in a relatively small and selective group of surviving patients, we consider the results a relevant indication, because they are similar to studies on the quality of life after a total laryngopharyngectomy [7, 24]. However, to fully appreciate the quality of life in this selective group of patients, a prospective study in a larger population is necessary. This would most likely require a multicenter study to include an adequate number of patients.

\section{Conclusion}

Total laryngopharyngectomy with circumferential reconstruction of the pharynx is generally indicated for a patient population with poor prognosis, and is associated with significant morbidity, high complication rates and lengthy rehabilitation. However, if patients do survive their disease, a good long-term quality of life can be expected. Good preoperative counseling of patients is, therefore, essential.

Conflict of interest statement The authors declare that they have no conflict of interest.

Open Access This article is distributed under the terms of the Creative Commons Attribution Noncommercial License which permits any noncommercial use, distribution, and reproduction in any medium, provided the original author(s) and source are credited.

\section{References}

1. Sewnaik A, Hoorweg JJ, Knegt PP et al (2005) Treatment of hypopharyngeal carcinoma: analysis of nationwide study in the Netherlands over a 10-year period. Clin Otolaryngol 30(1):52-57

2. Kim S, Wu HG, Heo DS et al (2001) Advanced hypopharyngeal carcinoma treatment results according to treatment modalities. Head Neck 23(9):713-717

3. Boscolo-Rizzo P, Maronato F, Marchiori C et al (2008) Longterm quality of life after total laryngectomy and postoperative radiotherapy versus concurrent chemoradiotherapy for laryngeal preservation. Laryngoscope 118(2):300-306

4. Pignon JP, Bourhis J, Domenge C et al (2000) Chemotherapy added to locoregional treatment for head and neck squamous-cell 
carcinoma: three meta-analyses of updated individual data. MACH-NC Collaborative Group. Meta-Analysis of Chemotherapy on Head and Neck Cancer. Lancet 355(9208):949-955

5. Triboulet JP, Mariette C, Chevalier D et al (2001) Surgical management of carcinoma of the hypopharynx and cervical esophagus: analysis of 209 cases. Arch Surg 136(10):11641170

6. Pauli SA, Vervloessem D, Vannieuwenhuyse PE et al (2006) Free jejunal autograft reconstruction after total pharyngolaryngeal resection. Int Surg 91(4):201-206

7. Hanson RP, Chow TK, Feehan E et al (2007) Analysis of functional results and quality of life following free jejunal flaps for reconstruction after upper aerodigestive neoplastic resection: the St James's experience. J Plast Reconstr Aesthet Surg 60(6):577582

8. Clark JR, Gilbert R, Irish J et al (2006) Morbidity after flap reconstruction of hypopharyngeal defects. Laryngoscope 116(2):173-181

9. Scharpf J, Esclamado RM (2005) Analysis of recurrence and survival after hypopharyngeal ablative surgery with radial forearm free flap reconstruction. Laryngoscope 115(3):429-432

10. Kolh P, Honore P, Degauque C et al (2000) Early stage results after oesophageal resection for malignancy-colon interposition vs. gastric pull-up. Eur J Cardiothorac Surg 18(3):293-300

11. Aaronson NK, Ahmedzai S, Bergman B et al (1993) The European Organization for Research and Treatment of Cancer QLQC30: a quality-of-life instrument for use in international clinical trials in oncology. J Natl Cancer Inst 85(5):365-376

12. Bjordal K, Hammerlid E, Ahlner-Elmqvist M et al (1999) Quality of life in head and neck cancer patients: validation of the European Organization for Research and Treatment of Cancer Quality of Life Questionnaire-H\&N35. J Clin Oncol 17(3): 1008-1019

13. Disa JJ, Pusic AL, Mehrara BJ (2006) Reconstruction of the hypopharynx with the free jejunum transfer. J Surg Oncol 94(6): 466-470

14. Lont HE, van Lanschot JJ, Hop WC et al (1994) Reconstruction of the digestive tract using a free small intestine interpositional graft following total larynx-pharynx extirpation. Follow-up results. Ned Tijdschr Geneeskd 138(26):1317-1321

15. Eckardt A, Meyer A, Laas U et al (2007) Reconstruction of defects in the head and neck with free flaps: 20 years experience. Br J Oral Maxillofac Surg 45(1):11-15

16. Nyquist GG, Hier MP, Dionisopoulos T, Black MJ (2006) Stricture associated with primary tracheoesophageal puncture after pharyngolaryngectomy and free jejunal interposition. Head Neck 28(3):205-209

17. Keereweer S, Sewnaik A, Kerrebijn JD, et al. (2009) Dysphagia after an L-shaped reconstruction technique of the free jejunum graft. J Plast Reconstr Aesthet Surg. 5 Dec 2009 (Epub ahead of print)

18. Murray DJ, Novak CB, Neligan PC (2008) Fasciocutaneous free flaps in pharyngolaryngo-oesophageal reconstruction: a critical review of the literature. J Plast Reconstr Aesthet Surg 61(10):1148-1156

19. Yu P, Lewin JS, Reece GP et al (2006) Comparison of clinical and functional outcomes and hospital costs following pharyngoesophageal reconstruction with the anterolateral thigh free flap versus the jejunal flap. Plast Reconstr Surg 117(3):968-974

20. Boscolo-Rizzo P, Zanetti F, Carpene S et al (2008) Long-term results with tracheoesophageal voice prosthesis: primary versus secondary TEP. Eur Arch Otorhinolaryngol 265(1):73-77

21. Lewin JS, Barringer DA, May AH et al (2005) Functional outcomes after circumferential pharyngoesophageal reconstruction. Laryngoscope 115(7):1266-1271

22. Oniscu GC, Walker WS, Sanderson R (2001) Functional results following pharyngolaryngooesophagectomy with free jejunal graft reconstruction. Eur J Cardiothorac Surg 19(4):406-410

23. Schilling MK, Eichenberger M, Maurer CA et al (2002) Longterm survival of patients with stage IV hypopharyngeal cancer: impact of fundus rotation gastroplasty. World J Surg 26(5):561565

24. Sewnaik A, van den Brink JL, Wieringa MH et al (2005) Surgery for recurrent laryngeal carcinoma after radiotherapy: partial laryngectomy or total laryngectomy for a better quality of life? Otolaryngol Head Neck Surg 132(1):95-98 